\title{
Clinical Study \\ Noncleft Velopharyngeal Insufficiency: Etiology and Need For Surgical Treatment
}

\author{
Steven Goudy, ${ }^{1}$ Christopher Ingraham, ${ }^{2}$ and John Canady ${ }^{3}$ \\ ${ }^{1}$ Department of Otolaryngology, Vanderbilt University, Nashville, TN, USA \\ ${ }^{2}$ Department of Radiology, University of Washington, Seattle, WA, USA \\ ${ }^{3}$ Department of Otolaryngology Head and Neck Surgery, University of Iowa, Iowa City, IA, USA
}

Correspondence should be addressed to Steven Goudy, steven.goudy@vanderbilt.edu

Received 28 November 2011; Accepted 23 January 2012

Academic Editor: James Brookes

Copyright (C) 2012 Steven Goudy et al. This is an open access article distributed under the Creative Commons Attribution License, which permits unrestricted use, distribution, and reproduction in any medium, provided the original work is properly cited.

\begin{abstract}
Objective. Velopharyngeal insufficiency (VPI) occurs frequently in cleft palate patients. VPI also occurs in patients without cleft palate, but little is known about this patient population and this presents a diagnostic dilemma. Our goal is to review the etiology of noncleft VPI and the surgical treatment involved. Design/Patients. A retrospective review of VPI patients from 1990 to 2005. Demographic, genetic, speech, and surgical data were collected. We compared the need for surgery and outcomes data between noncleft and cleft VPI patients using a Student's $t$-test. Results. We identified 43 patients with noncleft VPI, of which 24 were females and 19 were males. The average age at presentation of noncleft VPI was 9.6 years (range 4.5-21). The average patient age at the time of study was 13.4 years. The etiology of VPI in these noncleft patients was neurologic dysfunction $44 \%$, syndrome-associated $35 \%$, postadenotonsillectomy $7 \%$, and multiple causes $14 \%$. The need for surgical intervention in the noncleft VPI group was $37 \%$ (15/43) compared to the cleft palate controls, which was $27 \%(12 / 43)$. There was not a statistical difference between these two groups $(P>0.5)$. Conclusion. Noncleft VPI often occurs in patients who have underlying neurologic disorders or have syndromes. The rate of speech surgery to address VPI is similar to that of cleft palate patients. We propose that newly diagnosed noncleft VPI patients should undergo a thorough neurologic and genetic evaluation prior to surgery.
\end{abstract}

\section{Introduction}

The velopharyngeal complex closes against the posterior pharyngeal wall, to separate the oral cavity from the nasal cavity. Inability of the velopharyngeal complex to close leads to nasal air emissions and hypernasal speech. The occurrence of velopharyngeal insufficiency (VPI) in the absence of cleft palate formation has been reported many times in the literature. VPI is a recognized complication of adenoidectomy [1-8]. Moreover, patients with velocardiofacial syndrome (VCFS) also have VPI without cleft palate $[5,9,10]$.

Individuals with suspected VPI are best evaluated by a team of professionals specializing in VPI and speech characteristics associated with cleft palate. Initially, each of these patients is evaluated by a speech pathologist to assess the patient's speech patterns, nasality, and articulation disorders [11]. From there, they will undergo evaluation of their velopharyngeal port closure using multiview fluoroscopy and/or video nasoendoscopy $[12,13]$.
Once the diagnosis of VPI has been made a trial of speech therapy is often used to improve the intelligibility of speech. If speech remains unintelligible and/or VPI is a significant component of the speech pattern, surgical intervention is often needed. The type of surgery required is often based on the video nasoendoscopy [14]. The outcomes after speech surgery may vary based on the type of surgery performed and underlying medical diagnosis, especially in patients with VCFS $[15,16]$. We hypothesize that patients with noncleft VPI require similar surgical management and have similar speech outcomes compared to cleft palate patients.

\section{Materials and Methods}

After obtaining IRB approval, a retrospective review of patients charts diagnosed with VPI who did and did not have a cleft palate was performed. Patients with VPI treated at the University of Iowa Hospitals and Clinics from 1990 
to 2005 were reviewed. Demographic, genetic, speech, and surgical data were collected. For each noncleft VPI patient we identified an age-and-sex matched control patient with a cleft palate. We compared the outcomes data using a Student's t-test. Briefly, each patient was evaluated according to the protocol by Dailey et al. for hyper and hyponasality ( $1=$ normal, $2=$ mild, $3=$ mild-moderate, $4=$ moderate, $5=$ moderate-severe, 6 = severe) and underwent video nasal endoscopy [17]. Based on these findings, each patient was assessed to have a competent, marginally competent, or incompetent velopharyngeal closure. Patients judged to have marginally competent or incompetent velopharyngeal closure underwent surgical management of their VPI based on their port closure characteristics.

\section{Results}

We identified 43 patients who had noncleft VPI. There were 24 females and 19 males found. The average age at the diagnosis of noncleft VPI was 9.6 years (range $4.5-21$ ). The average age of the patient in the study was 13.4 years. The etiology of VPI in these noncleft patients was postadenotonsillectomy $7 \%$, neurologic dysfunction $44 \%$, syndromeassociated $35 \%$, and unknown causes $14 \%$.

The neurologic dysfunction group comprised the largest portion of the noncleft VPI group (44\%). There were a wide spectrum of disorders included in this group detailed in Table 1, which includes posttraumatic brain injury, developmental delay, and cerebral palsy.

The occurrence of a syndrome in the noncleft patients with VPI was 35\% and is shown in Table 2, which includes VCFS, Turner syndrome, and VATER syndrome. Of note, a new diagnosis of VCFS was made in 6 of our patients. The remaining group of patients did not have any definable risk factors for VPI.

The need for surgical intervention in the noncleft VPI group was 37\% (15/43) compared to the cleft palate controls, which was $27 \%$ (12/43). In noncleft VPI patients, 10 pharyngeal flaps, 4 double-opposing $Z$ plasties, and 1 sphincter pharyngoplasty were performed. In the cleft palate control group 11 pharyngeal flaps and 1 double-opposing $\mathrm{Z}$ plasty were performed. There was not a statistical difference in the number of VPI surgeries between these two groups $(P>0.5)$.

\section{Discussion}

It is clear that noncleft VPI occurs in an assortment of anatomic, neurologic, postsurgical, and syndromic patients. A standard speech assessment is critical for making the diagnosis. The noncleft VPI patients were identified at a much later age, 9.6 years, than children with cleft palates. This is likely due to the speech screening that occurs in most cleft palate teams that identifies VPI earlier in children with cleft palate.

Surgical intervention was required in 37\% of noncleft VPI patients which is lower than what is reported in other studies $[2,3,6]$. The rate of VPI surgery did not statistically
TABLE 1: Neurologic causes of noncleft VPI.

\begin{tabular}{lc}
\hline Posttraumatic brain injury & $N=2$ \\
Developmental delay & $N=2$ \\
Cerebral palsy & $N=1$ \\
Myasthenia gravis & $N=1$ \\
Associated speech delay & $N=3$ \\
Undiagnosed neurologic condition & $N=10$ \\
\hline
\end{tabular}

TABLE 2: Syndromic causes of noncleft VPI.

\begin{tabular}{ll}
\hline Velocardiofacial syndrome & $N=7$ \\
Klippel-Feil syndrome & $N=1$ \\
Epidermal-Nevus syndrome & $N=1$ \\
Alagille syndrome & $N=1$ \\
Turner syndrome & $N=1$ \\
VATER syndrome & $N=1$ \\
Unrecognized syndrome & $N=1$ \\
\hline
\end{tabular}

differ between noncleft and cleft palate patients. This suggests that noncleft VPI can be treated similar to cleft palate patients with initial speech therapy to improve articulation. However unresolved VPI required surgical intervention in more than a third of patients.

It is important to note that an etiology of the VPI could be specifically identified in $86 \%$ of patients. Many of the patients had underlying neurological diagnoses or syndromes suggesting that altered neuromuscular control is associated with noncleft VPI. Newly diagnosed VCFS was identified in $16 \%$ of our patients, the importance of genetic evaluation has been highlighted by other authors [5].

\section{Conclusion}

Noncleft VPI occurs in a diverse group of patients. The majority of these patients will not require surgical intervention. Each patient with noncleft VPI should be carefully screened for the etiology of their VPI because only 14\% will have an unknown cause. The need for VPI surgery in noncleft patients is not different than cleft palate patients.

\section{References}

[1] M. A. Witzel, R. H. Rich, F. Margar-Bacal, and C. Cox, "Velopharyngeal insufficiency after adenoidectomy: an 8-year review," International Journal of Pediatric Otorhinolaryngology, vol. 11, no. 1, pp. 15-20, 1986.

[2] K. J. Stewart, T. Ahmad, A. C. H. Watson, and R. E. Razzell, "Altered speech following adenoidectomy: a 20 year experience," British Journal of Plastic Surgery, vol. 55, no. 6, pp. 469-473, 2002.

[3] N. C. Saunders, B. E. J. Hartley, D. Sell, and B. Sommerlad, "Velopharyngeal insufficiency following adenoidectomy," Clinical Otolaryngology and Allied Sciences, vol. 29, no. 6, pp. 686-688, 2004. 
[4] Y. F. Ren, A. Isberg, and G. Henningsson, "Velopharyngeal incompetence and persistent hypernasality after adenoidectomy in children without palatal defect," Cleft PalateCraniofacial Journal, vol. 32, no. 6, pp. 476-482, 1995.

[5] J. A. Perkins, K. Sie, and S. Gray, "Presence of 22q11 deletion in postadenoidectomy velopharyngeal insufficiency," Archives of Otolaryngology, Head and Neck Surgery, vol. 126, no. 5, pp. 645-648, 2000.

[6] D. B. Fernandas, A. O. Grobbelaar, D. A. Hudson, and R. Lentin, "Velopharyngeal incompetence after adenotonsillectomy in non-cleft patients," British Journal of Oral and Maxillofacial Surgery, vol. 34, no. 5, pp. 364-367, 1996.

[7] L. L. D’Antonio, L. S. Snyder, and S. Samadani, "Tonsillectomy in children with or at risk for velopharyngeal insufficiency: effects on speech," Otolaryngology, Head and Neck Surgery, vol. 115, no. 4, pp. 319-323, 1996.

[8] C. B. Croft, R. J. Shprintzen, and R. J. Ruben, "Hypernasal speech following adenotonsillectomy," Otolaryngology, Head and Neck Surgery, vol. 89, no. 2, pp. 179-188, 1981.

[9] J. G. Boorman, S. Varma, and C. Mackie Ogilvie, "Velopharyngeal incompetence and chromosome 22q11 deletion," Lancet, vol. 357, no. 9258, p. 774, 2001.

[10] N. H. Robin and R. J. Shprintzen, "Defining the clinical spectrum of deletion 22q11.2," Journal of Pediatrics, vol. 147, no. 1, pp. 90-96, 2005.

[11] L. E. Eblen, K. C.Y. Sie, and L. T. Furlow, "Perceptual and instrumental assessment of velopharyngeal insufficiency," Plastic and Reconstructive Surgery, vol. 109, no. 7, pp. 25892590, 2002.

[12] R. J. Shprintzen and K. J. Golding-Kushner, "Evaluation of velopharyngeal insufficiency," Otolaryngologic Clinics of North America, vol. 22, no. 3, pp. 519-536, 1989.

[13] D. J. Lam, J. R. Starr, J. A. Perkins et al., "A comparison of nasendoscopy and multiview videofluoroscopy in assessing velopharyngeal insufficiency," Otolaryngology, Head and Neck Surgery, vol. 134, no. 3, pp. 394-402, 2006.

[14] R. J. Shprintzen, M. L. Lewin, and C. B. Croft, "A comprehensive study of pharyngeal flap surgery: tailor made flaps," Cleft Palate Journal, vol. 16, no. 1, pp. 46-55, 1979.

[15] A. Losken, J. K. Williams, F. D. Burstein, D. N. Malick, and J. E. Riski, "Surgical correction of velopharyngeal insufficiency in children with velocardiofacial syndrome," Plastic and Reconstructive Surgery, vol. 117, no. 5, pp. 1493-1498, 2006.

[16] L. M. De Serres, F. W. B. Deleyiannis, L. E. Eblen, J. S. Gruss, M. A. Richardson, and K. C. Y. Sie, "Results with sphincter pharyngoplasty and pharyngeal flap," International Journal of Pediatric Otorhinolaryngology, vol. 48, no. 1, pp. 17-25, 1999.

[17] S. A. Dailey, M. P. Karnell, L. H. Karnell, and J. W. Canady, "Comparison of resonance outcomes after pharyngeal flap and furlow double-opposing Z-plasty for surgical management of velopharyngeal incompetence," Cleft Palate-Craniofacial Journal, vol. 43, no. 1, pp. 38-43, 2006. 


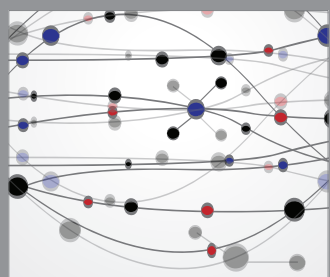

The Scientific World Journal
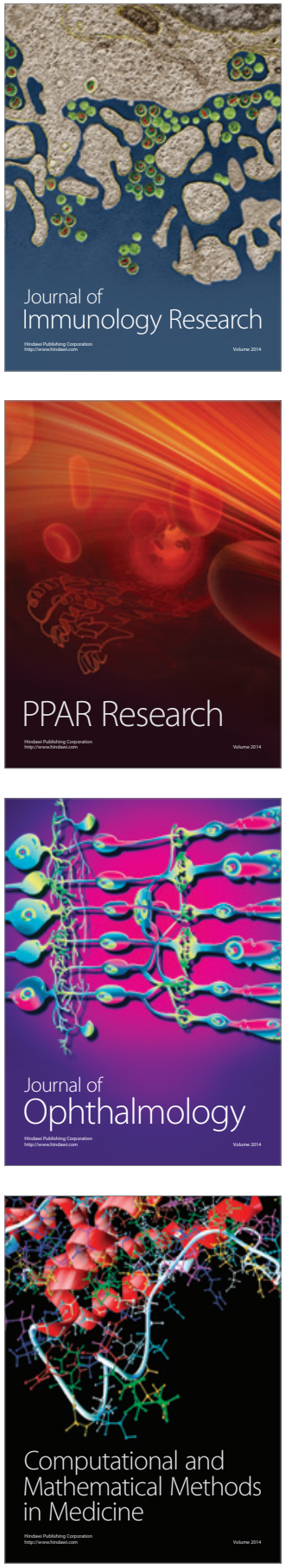

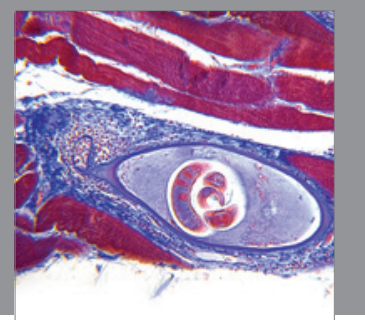

Gastroenterology

Research and Practice
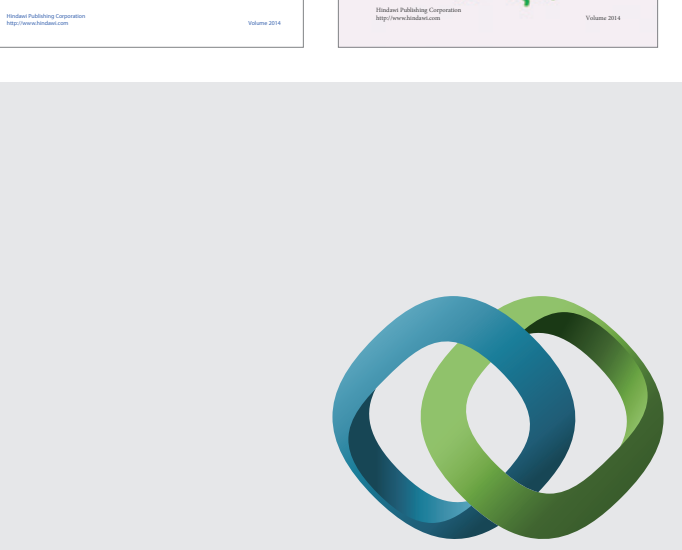

\section{Hindawi}

Submit your manuscripts at

http://www.hindawi.com
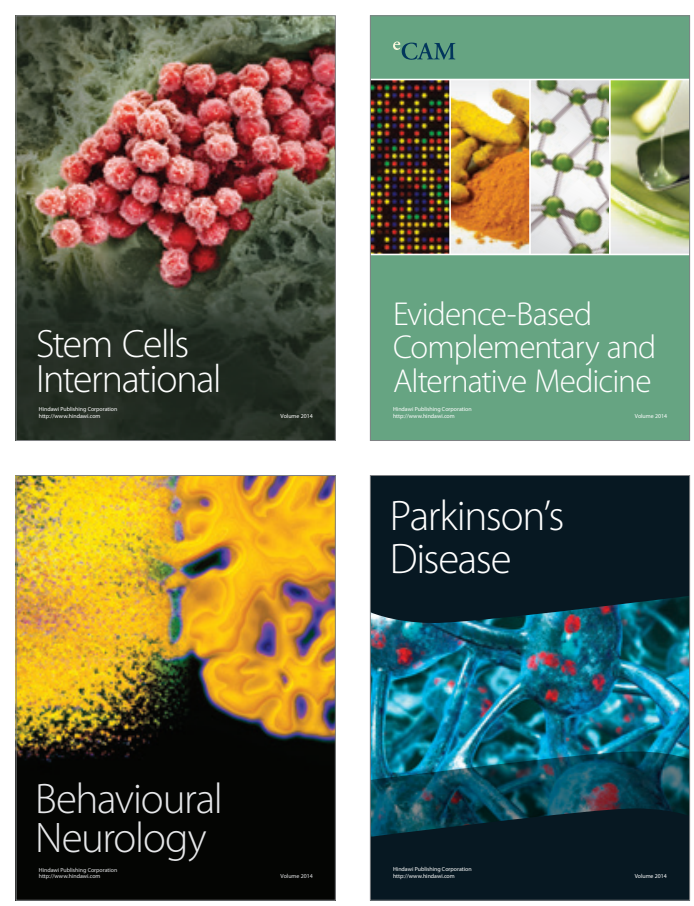

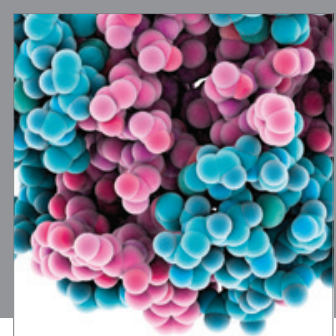

Journal of
Diabetes Research

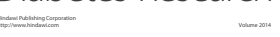

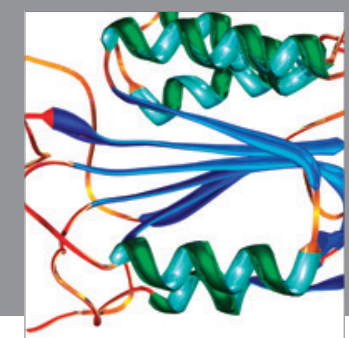

Disease Markers
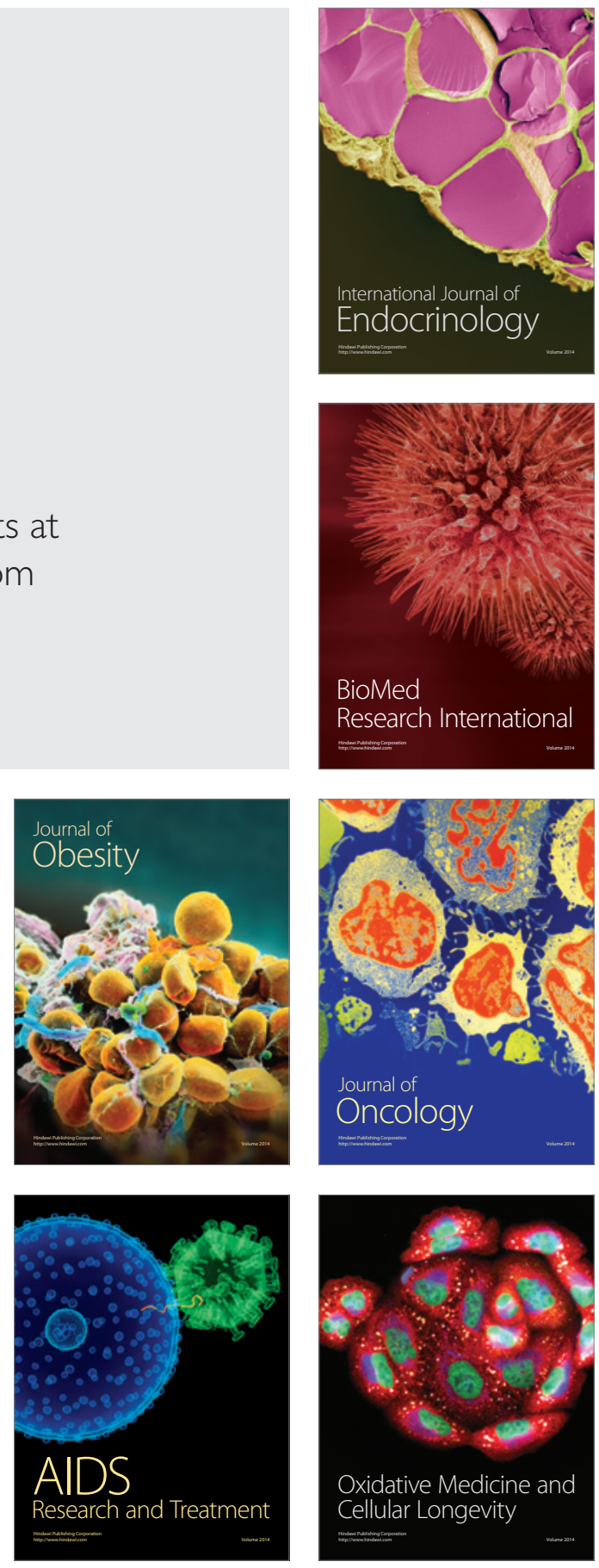